\title{
Premixed Edge-Flames under Transverse Enthalpy Gradients
}

\author{
J. DAOU and M. MATALON* \\ McCormick School of Engineering and Applied Science, Engineering Sciences and Applied Mathematics, \\ Northwestern University, 2145 Sheridan Road, Evanston, IL 60208, USA
}

and

\author{
A. LIÑÁN \\ Dpto. Motopropulsion y Termofuidodinamica, UPM, E.T.S.I Aeronáuticos, Pza. Cardenal Cisneros 3, 28040 \\ Madrid, Spain
}

\begin{abstract}
We describe flame propagation between two opposed reactive streams which may differ in their composition and temperature. A two-dimensional counterflow configuration and an irreversible Arrhenius reaction are adopted, along with the constant density approximation. Attention is focused on the influence of two nondimensional parameters. The first one, denoted by $\gamma$, represents the difference in the enthalpy of the feed streams. The second one, $\epsilon$, quantifies the ratio between the characteristic chemical time and the strain time. After a general formulation of the problem, we begin by an analysis of the one-dimensional case consisting of two parallel planar flames of unequal strength. The flames behavior is described analytically and numerically. In particular, two extinction regimes are identified: for values of $\gamma$ smaller than a critical value $\gamma^{*}$, the flames extinguish by quenching against each other at the stagnation plane; for $\gamma>\gamma^{*}$ they extinguish while at a finite distance from each other which increases with $\gamma$. These behaviors are similar to those, known in the literature, associated with the influence of Lewis numbers on the extinction of twin-flames. We then describe the propagation of two-dimensional flame fronts along the stagnation line, in a direction perpendicular to the plane of strain. The flame front is thus curved under the combined effects of the flow field and the transverse enthalpy gradient in the frozen mixture ahead of it; far behind the state of the gas is that of the pair of flat flames introduced above. The problem is studied numerically and complemented by an analytical description of the fast-chemistry situations corresponding to small values of $\epsilon$. In particular we describe, for different fixed values of $\gamma$, the evolution of ignition fronts, characterized by a positive propagation speed, to extinction fronts, characterized by negative speeds, as $\epsilon$ is increased. In addition to the marked change in the flame shape, the most noticeable effect of an increase in $\gamma$ is the decrease in the propagation speed of the flame front. These effects are associated with the increased front curvature for higher values of $\gamma$, along with a shift of the front leading edge towards the stream with higher enthalpy. (C) 2000 by The Combustion Institute
\end{abstract}

\section{INTRODUCTION}

In combustion applications flames often propagate in combustible mixtures in which the state of the fresh reactive gas is nonuniform. An important class of nonuniformities is one associated with enthalpy gradients. Such gradients result from variations in the composition of the reactants and their temperature. Examples include flame spread over solid or liquid fuel surfaces, flame propagation in mixing layers, deflagration towards or along walls, and ignition in mixtures with spatially changing equivalence ratio. Because enthalpy variations directly affect the flame temperature, their influence on the rates of the thermally sensitive chemical reactions is considerable, and can have significant

Corresponding author. E-mail: matalon@nwu.edu consequences on fuel consumption, flame quenching, and emission of chemical pollutants. Therefore, combustion in nonuniform mixtures is an important problem, both from the fundamental and practical viewpoints. The problem, in general, is complicated since enthalpy gradients may vary spatially and temporally across the combustion field, and are typically coupled to complex fluid flows. In addition, the scales involved may span a wide spectrum in the various situations of relevance.

When the compositions in the fresh mixture vary from lean to rich conditions, such as in mixing layers of initially nonpremixed reactants or when holes are created in a diffusion flame, a tribrachial or triple flame is formed. Such a flame consists of three branches: a lean premixed branch, a rich premixed branch, and a trailing diffusion flame. Early experimental ob- 
servation of this structure was made by Phillips [1], and an early analytical description appears in Ohki and Tsuge [2]. Over the last 10 years there has been a strong interest in triple flames and substantial work on these structures and their propagation regimes was undertaken by Dold [3, 4], and others [5-10]. When the fresh mixture is off-stoichiometric, we are dealing with premixed systems. It has been shown recently that triple flame like structures, termed edge-flames by Buckmaster [11], are also possible in premixed systems [11-14]. In particular, configurations in which the edge flame is supported by a counterflow of fresh/fresh or fresh/ inert gas mixture were investigated. Complementary aspects on premixed edge flames, including nonunity Lewis number effects, have been reported in [15]. On the experimental side, interesting findings on edge-flames, both in premixed and nonpremixed counterflow situations, have been presented by Ronney and coworkers, $[10,16]$. They describe, in particular, stationary edges under spatially varying strain.

It should be noted that in studies of premixed combustion the effects of nonuniformities in the mixture's composition and temperature have been seldom taken into account. In fact, the uniformity of the fresh reactive mixture appears as a common basic assumption in theoretical work on stretched flames; see, for example, [17, 18]. Investigations accounting for enthalpy gradients exist, however; for example, enthalpy variations in the direction of flame propagation are considered in different works mainly related to the stratified charge combustion engine [19, 20 ], or to flames approaching walls [21].

The specific problem under consideration is concerned with the twin-flame counterflow configuration but with the opposed combustible streams differing in their temperature and composition. This provides a simple setting for our numerical and analytical investigation of the effects of enthalpy nonuniformities on flame propagation. Two types of solutions are described, corresponding respectively to two-dimensional flame-edges propagating in a direction perpendicular to the plane of strain, and to the one-dimensional pair of planar flames of unequal strength which constitute their trailing branches. The study of the two-dimensional fronts provides insight, in particular, into the

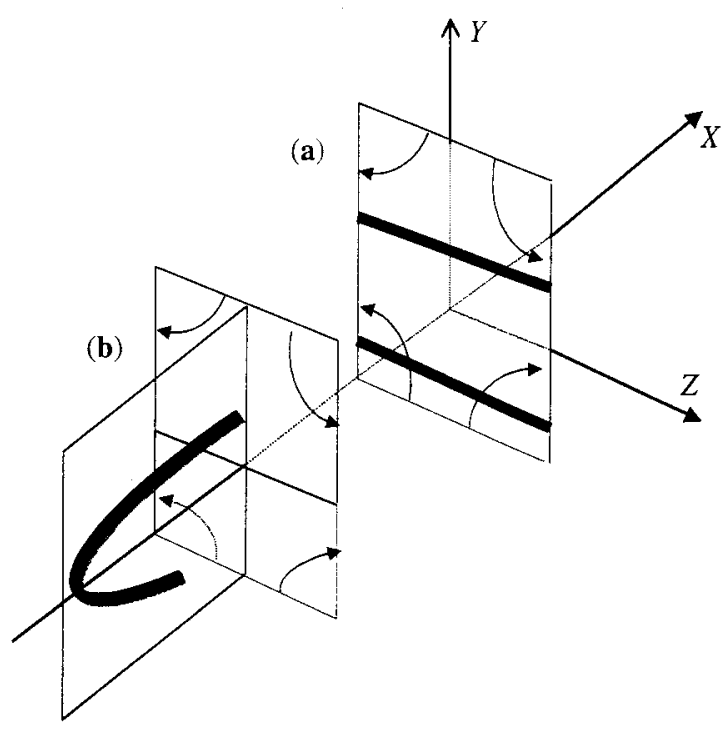

Fig. 1. (a) Twin flames (the thick lines parallel to the $Z$-axis) in a counterflow configuration. The flow is twodimensional with the $Y$ and $Z$ components given by $v_{Y}=$ $-a Y$ and $v_{Z}=a Z$, where $a$ is the strain rate. (b) A two-dimensional edge flame; the flame shape is independent of $Z$ and the propagation is along the $X$-axis. The trailing wings of the edge flame, for large positive $X$, coincide with the twin flames depicted in (a).

evolution of ignition fronts, characterized by a positive propagation speed, and extinction fronts, characterized by a negative propagation speed, as argued in [15].

We shall begin by a general formulation in the frame of the constant density approximation. The planar-flames case is then addressed, for which analytical and numerical descriptions are given. This is followed by the 2D case which is studied numerically, and analytically in the fast chemistry limit.

\section{FORMULATION}

The configuration under consideration is that of two opposed streams of the same reactive mixture, differing in their initial composition and temperature and impinging against each other as sketched in Fig. 1. We shall adopt the thermodiffusive model of constant density and constant transport properties. The flow field is described by $v_{X}=0, v_{Y}=-a Y$, and $v_{Z}=a Z$, where $a$ is the strain rate; far to the right along the $X$-direction the field supports a pair of 
planar flames occupying different locations relative to the stagnation plane, provided that the strain rate is below an extinction value; far to the left the mixture is chemically frozen. An edge flame, consisting of a curved front connecting the pair of planar flames, can either spread by moving in the negative $X$-direction, or retract moving in the positive $X$-direction. The temperature and composition fields are sought independent of the $Z$-coordinate so that a sketch can be drawn in the $(X, Y)$ plane as in Fig. 1b. We shall use a frame of reference attached to the flame so that the profiles are also time-independent and the velocity field $\mathbf{v}$ is given by $\mathbf{v}=(\tilde{U},-a Y, a Z)$, where $\tilde{U}$ is the propagation speed of the flame front. $\tilde{U}$ will be positive when the flame advances in the negative $X$-direction, corresponding to an ignition front, and negative for retreating or extinction fronts.

The combustion will be represented by a one-step irreversible Arrhenius reaction which consumes the fuel, considered to be deficient, at a rate

$\tilde{\omega}=\rho Y_{F} B \exp (-E / R T)$,

where $B, Y_{F}, E, R$ represent respectively the preexponential factor, the mass fraction of fuel, the activation energy, and the universal gas constant.

The conservation equations are

$\tilde{U} \frac{\partial Y_{F}}{\partial X}-a Y \frac{\partial Y_{F}}{\partial Y}=D_{F} \Delta Y_{F}-\frac{\tilde{\omega}}{\rho}$

$\tilde{U} \frac{\partial T}{\partial X}-a Y \frac{\partial T}{\partial Y}=D_{T} \Delta T+\frac{q}{c_{p}} \frac{\tilde{\omega}}{\rho}$,

where $D_{F}$ is the fuel diffusion coefficient, $D_{T}$ and $c_{p}$ the thermal diffusivity and heat capacity, and $q$ the heat released per unit mass of fuel.

We shall use the conditions prevailing in the upper stream, at $Y=+\infty$, to define a reference flame speed $S_{L}^{0}=\sqrt{2 \beta^{-2} D_{T} B \exp \left(-E / R T_{a d}\right)}$, and to introduce normalized fuel mass fraction and temperature by

$y_{F}=\frac{Y_{F}}{Y_{u}}$ and $\theta=\frac{T-T_{u}}{T_{a d}-T_{u}}$

Here $T_{a d} \equiv T_{u}+q Y_{u} / c_{p}$ is the adiabatic flame temperature, and $\beta \equiv E\left(T_{a d}-T_{u}\right) / R T_{a d}^{2}$ the
Zeldovich number. The expression of $S_{L}^{0}$ corresponds to the asymptotic approximation for $\beta \gg 1$ of the burning speed of a planar flame with the Lewis number, $L e=D_{T} / D_{F}$, equal to unity.

When $S_{L}^{0}$ is selected as a unit speed, and the convective-diffusive thickness $L=\sqrt{2 D_{T} / a}$ as a unit length, the governing equations can be written as

$U \frac{\partial y_{F}}{\partial x}-2 \epsilon y \frac{\partial y_{F}}{\partial y}=\frac{\epsilon}{L e_{F}} \Delta y_{F}-\epsilon^{-1} \omega$

$U \frac{\partial \theta}{\partial x}-2 \epsilon y \frac{\partial \theta}{\partial y}=\epsilon \Delta \theta+\epsilon^{-1} \omega$

Here

$\epsilon \equiv \frac{l_{F l}^{0}}{L}=\frac{l_{F l}^{0}}{\sqrt{2 D_{T} / a}}$

is the ratio of the premixed flame thickness, $l_{F l}^{0}=D_{T} / S_{L}^{0}$, to the reference length $L$; it is related to the Damköhler number, Da, by $\epsilon^{-2}=$ $\mathrm{Da}$ if $\mathrm{Da}$ is defined as the ratio of the flow time, $2 a^{-1}$, to the chemical reaction time $l_{F l}^{0}{ }^{2} / D_{T}$. The scaled propagation speed $U=\tilde{U} / S_{L}^{0}$ is an eigenvalue, and its determination is the goal of the analysis. The nondimensional reaction rate $\omega$ is given by

$\omega=\frac{\beta^{2}}{2} y_{F} \exp \left\{\frac{\beta(\theta-1)}{1+\alpha_{h}(\theta-1)}\right\}$,

where $\alpha_{h} \equiv\left(T_{a d}-T_{u}\right) / T_{a d}$ is the heat-release parameter.

The boundary conditions are

$\theta=0, \quad y_{F}=1$ as $y \rightarrow \infty$

$\theta=-\Delta_{T}, \quad y_{F}=1-\Delta_{F}$ as $y \rightarrow-\infty$

corresponding to the conditions in the feed streams,

$y_{F}=1-\frac{\Delta_{F}}{2} \operatorname{erfc}\left(y \sqrt{L e_{F}}\right)$,

$\theta=-\frac{\Delta_{T}}{2} \operatorname{erfc}(y)$ for $x \rightarrow-\infty$

corresponding to the frozen conditions, and

$\frac{\partial y_{F}}{\partial x}=\frac{\partial \theta}{\partial x}=0$ for $x \rightarrow \infty$ 
since the profiles are expected to be $x$-independent far downstream. The parameters $\Delta_{F}$ and $\Delta_{T}$ are defined by

$$
\Delta_{F}=\frac{Y_{u}-Y_{u}^{-}}{Y_{u}} \text { and } \Delta_{T}=\frac{T_{u}-T_{u}^{-}}{q Y_{u} / c_{p}}
$$

At this stage, the problem formulation is complete and is suitable for a numerical treatment in which $\beta$ is finite, such as the one to be given later.

Before addressing the propagating edgeflame problem, which corresponds to solutions of the two-dimensional problem we have just formulated, we shall give an analytic description of their trailing wings which far downstream constitute a pair of flat flames independent of $x$. The analytic predictions, valid in the asymptotic limit $\beta \rightarrow \infty$, will be followed by the presentation of numerical calculations with finite $\beta$. Of course, the one-dimensional study under consideration is interesting by itself, since it depicts the interaction between two flat flames pushed against each other by a counterflow with different feed conditions.

\section{THE PLANAR FLAME FRONTS}

In this section we consider the limit

$\beta \rightarrow \infty$ while $\epsilon, \gamma_{F}, \gamma_{\theta} \sim O(1)$

Here the parameters $l_{F}, \gamma_{F}$ and $\gamma_{\theta}$ are defined by

$l_{F}=\beta\left(L e_{F}-1\right), \quad \gamma_{F}=\beta \Delta_{F}$ and $\gamma_{\theta}=\beta \Delta_{T}$

A reformulation of the problem in terms of the leading order temperature, $\theta^{0}$, and the excess enthalpy $h=\theta^{1}+y_{F}^{1}$ is then possible [22], where superscripts indicate orders of expansions in terms of $\beta^{-1}$.

More precisely, since $\beta \rightarrow \infty$, the reaction is confined to a pair of infinitely thin sheets on either side of which the equations

$$
\begin{aligned}
& \frac{d^{2} \theta^{0}}{d y^{2}}+2 y \frac{d \theta^{0}}{d y}=0 \\
& \frac{d^{2} h}{d y^{2}}+2 y \frac{d h}{d y}=-l_{F} \frac{d^{2} \theta^{0}}{d y^{2}},
\end{aligned}
$$

must be satisfied along with the boundary conditions

$$
\begin{array}{ll}
\theta^{0}=0, & h=0 \text { as } y \rightarrow \infty \\
\theta^{0}=0, & h=-\gamma \text { as } y \rightarrow-\infty
\end{array}
$$

Note that in the distinguished limit considered, the differences in the composition and temperature of the incoming streams are expressed by a single parameter

$\gamma=\gamma_{F}+\gamma_{\theta}$

which is positive, since we assume that the enthalpy of the upper stream is larger than that of the lower stream.

Finally at the reaction sheets, whose locations are denoted by $y_{m}$ and $y_{p}$ with $y_{p}>y_{m}$, the jump conditions to be satisfied are:

$\left[\theta^{0}\right]=[h]=0$

$-\frac{1}{l_{F}}\left[\frac{d h}{d y}\right]=\left[\frac{d \theta^{0}}{d y}\right]=-\epsilon^{-1} e^{\sigma / 2}$

Here $\sigma$ stands for the perturbations in the flames temperature, $\sigma_{m} \equiv h\left(y_{m}\right)$ and $\sigma_{p} \equiv$ $h\left(y_{p}\right)$. The squared bracket applied to any quantity denotes the jump; namely the value of this quantity at the burnt-gas side of the sheet minus its value at the unburnt side. The locations $y_{m}$ and $y_{p}$ are to be determined in terms of $\epsilon, \gamma$, and $l_{F}$, along with the corresponding burning rates per unit flame surface. The burning rates are given by $\mu_{m}=\exp \left(\sigma_{m} / 2\right)$ and $\mu_{p}=\exp \left(\sigma_{p} / 2\right)$. Using the boundary conditions (12) and the continuity of $\theta^{0}$ and $h$ across the reaction zones, solutions in the different regions delimited by the flames are given as follows.

Upper region $\left(y_{p}<y\right)$ :

$$
\theta^{0}=\frac{\operatorname{erfc}(y)}{\operatorname{erfc}\left(y_{p}\right)}
$$

$$
\begin{aligned}
h= & \sigma_{p} \frac{\operatorname{erfc}(y)}{\operatorname{erfc}\left(y_{p}\right)} \\
& +l_{F} \frac{y e^{-y^{2}} \operatorname{erfc}\left(y_{p}\right)-y_{p} e^{-y_{p}^{2}} \operatorname{erfc}(y)}{\sqrt{\pi} \operatorname{erfc}^{2}\left(y_{p}\right)}
\end{aligned}
$$


Intermediate region $\left(y_{m}<y<y_{p}\right)$ :

$\theta^{0}=1$

$h=\frac{\sigma_{p}\left\{\operatorname{erf}(y)-\operatorname{erf}\left(y_{m}\right)\right\}-\sigma_{m}\left\{\operatorname{erf}(y)-\operatorname{erf}\left(y_{p}\right)\right\}}{\operatorname{erf}\left(y_{p}\right)-\operatorname{erf}\left(y_{m}\right)}$

Lower region $\left(y<y_{m}\right)$ :

$$
\begin{aligned}
\theta^{0}= & \frac{1+\operatorname{erf}(y)}{1+\operatorname{erf}\left(y_{m}\right)} \\
h= & \left(\sigma_{m}+\gamma\right) \frac{1+\operatorname{erf}(y)}{1+\operatorname{erf}\left(y_{m}\right)} \\
& \quad+l_{F} \frac{y_{m}\{1+\operatorname{erf}(y)\} e^{-y_{m}^{2}}-\left\{1+\operatorname{erf}\left(y_{m}\right)\right\} y e^{-y^{2}}}{\sqrt{\pi}\left\{1+\operatorname{erf}\left(y_{m}\right)\right\}^{2}}
\end{aligned}
$$

The jump conditions then yield

$$
\begin{gathered}
\sigma_{p}=-\frac{\left(\sigma_{p}-\sigma_{m}\right) \operatorname{erfc}\left(y_{p}\right)}{\operatorname{erf}\left(y_{p}\right)-\operatorname{erf}\left(y_{m}\right)} \\
-\frac{l_{F}}{2}\left\{1+2 y_{p}^{2}-\frac{2 y_{p} e^{-y_{p}^{2}}}{\sqrt{\pi} \operatorname{erfc}\left(y_{p}\right)}\right\} \\
\boldsymbol{\epsilon}=\frac{\sqrt{\pi}}{2} \operatorname{erfc}\left(y_{p}\right) \exp \left(y_{p}^{2}+\sigma_{p} / 2\right) \\
\sigma_{m}=-\gamma+\frac{\left(\sigma_{p}-\sigma_{m}\right)\left(1+\operatorname{erf}\left(y_{m}\right)\right.}{\operatorname{erf}\left(y_{p}\right)-\operatorname{erf}\left(y_{m}\right)} \\
-\frac{l_{F}}{2}\left\{1+2 y_{m}^{2}+\frac{2 y_{m} e^{-y_{m}^{2}}}{\sqrt{\pi}\left\{1+\operatorname{erf}\left(y_{m}\right)\right\}}\right\} \\
\boldsymbol{\epsilon}=\frac{\sqrt{\pi}}{2}\left\{1+\operatorname{erf}\left(y_{m}\right)\right\} \exp \left(y_{m}^{2}+\sigma_{m} / 2\right)
\end{gathered}
$$

Solutions to the system of Equations 15 are simply determined in the two special cases corresponding to (i) $\gamma=0$ and (ii) $l_{F}=0$. In the former case, Lewis number effects can be studied in the symmetrical situation of twin-flames and could be found in the literature [22-24]. In the latter case, with which we shall be concerned, the role of unequal feed enthalpies can be investigated in the absence of differential diffusion.

The starting point for the discussion below is the system of equations

$$
\begin{aligned}
\sigma_{p} & =-\frac{\gamma}{2} \operatorname{erfc}\left(y_{p}\right) \\
\epsilon & =\frac{\sqrt{\pi}}{2} \operatorname{erfc}\left(y_{p}\right) \exp \left(y_{p}^{2}+\sigma_{p} / 2\right) \\
\sigma_{m} & =-\frac{\gamma}{2} \operatorname{erfc}\left(y_{m}\right) \\
\epsilon & =\frac{\sqrt{\pi}}{2}\left\{1+\operatorname{erf}\left(y_{m}\right)\right\} \exp \left(y_{m}^{2}+\sigma_{m} / 2\right),
\end{aligned}
$$

obtained after simple manipulations from (15) when $l_{F}=0$. The first two equations in (16) can be viewed as a parametric representation $\left\{\sigma_{p}\left(y_{p}\right), \epsilon\left(y_{p}\right)\right\}$ for the perturbation in the upper flame temperature $\sigma_{p}$ as a function of $\epsilon$. This yields a plot of the burning rate $\mu_{p}=$ $\exp \left(\sigma_{p} / 2\right)$ versus $\epsilon$. Similarly the last two equations provide a plot of $\sigma_{m}$ and the burning rate $\mu_{m}=\exp \left(\sigma_{m} / 2\right)$ of the lower flame versus $\epsilon$. In these parametric plots, the domain of variation of the parameters $y_{m}$ and $y_{p}$, representing the locations of the lower and upper flame, respectively, is of course restricted by the obvious requirement that $y_{m} \leq y_{p}$. The preceding inequality is strict, except if both $y_{p}$ and $y_{m}$ are equal to zero. In other words, the only location where the flames can touch, if any, is at the stagnation plane. This follows from (16), since the condition $\sigma_{m}=\sigma_{p}$, expressing the equality of the flames temperatures, must be satisfied along with the condition $y_{m}=y_{p}$ if the flames are to merge. It may be useful to point out that the preceding conclusions are drawn in the limit $\beta \rightarrow \infty$, for which the meaning and the locations of the reaction sheets are well defined. Nevertheless, the results to be derived in this limit, such as those concerning extinction given later, will still provide good approximations in situations where $\beta$ is finite but sufficiently large, as we shall verify using numerical calculations. We also note, for later reference, that as $\epsilon \rightarrow 0$ we have

$\sigma_{p} \rightarrow 0$ and $y_{p} \sim \frac{1}{2 \epsilon}$

$\sigma_{m} \rightarrow 0$ and $y_{m} \sim-\frac{1}{2 \epsilon} e^{-\gamma / 2}$ 


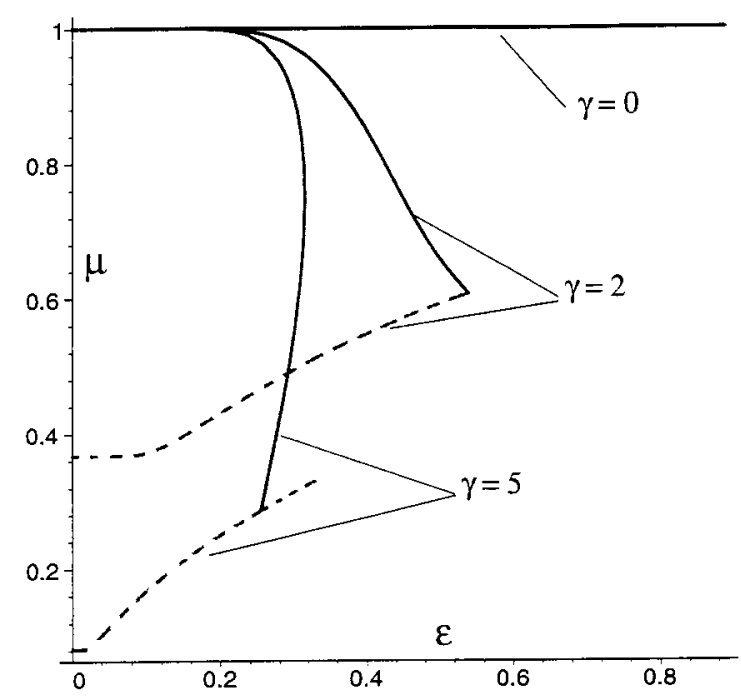

Fig. 2. The burning rates $\mu_{m}$ (dashed lines) and $\mu_{p}$ (solid lines) corresponding to the lower and upper flames, respectively, for three values of $\gamma$.

Our presentation of the results begins with Figs. 2 and 3 , where the burning rates $\left(\mu_{m}, \mu_{p}\right)$, and the flame locations, $\left(y_{m}, y_{p}\right)$, are plotted versus $\epsilon$ for three values of $\gamma$. For $\gamma=0$, the twin-flames are located symmetrically as expected, that is $y_{m}=-y_{p}$. Their burning rates are equal, $\mu_{m}=\mu_{p}$; in fact they are equal to unity in this particular case where both $\gamma$ and $l_{F}$

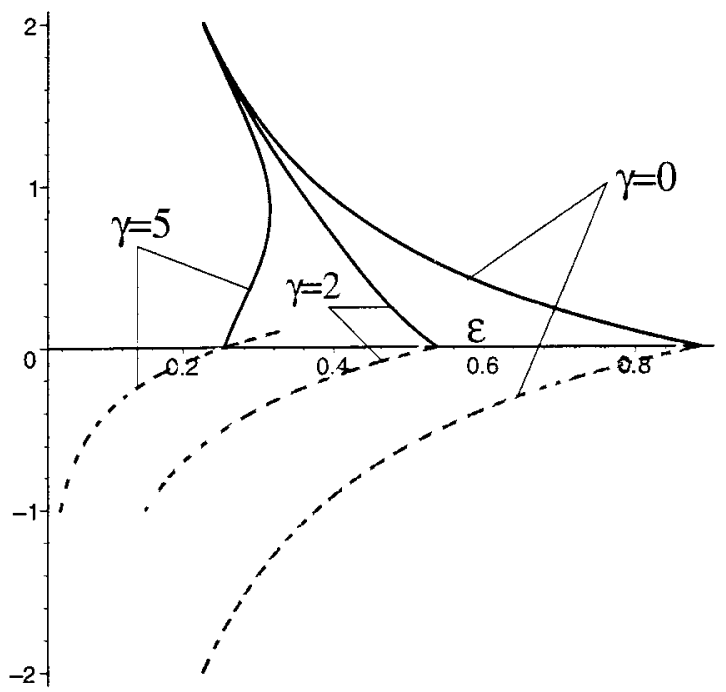

Fig. 3. The reaction-sheet locations $y_{m}$ (dashed lines) and $y_{p}$ (solid lines) corresponding to the lower and upper flame, respectively, for three values of $\gamma$, as in Fig. 2. are zero. As $\epsilon$ increases, the twin-flames approach each other until their reaction sheets meet at the stagnation plane, $y=0$. This happens at a value of $\epsilon$ beyond which no (burning) solution exists, and which may thus be identified with extinction. The trend is similar for $\gamma=2$, except that the flames are now asymmetrical; the upper flame being stronger than the lower one. Despite this asymmetry the flames eventually touch at $y=0$ as $\epsilon$ is increased. In contrast, for $\gamma$ larger than a critical value $\gamma^{*}$, a turning point appears in the curve of $y_{p}\left(\right.$ or $\left.\mu_{p}\right)$ vs. $\epsilon$, as illustrated for $\gamma=5$. Beyond the value of $\epsilon$ corresponding to the turning point, no burning solutions exist, so that this value may be used to define extinction. The critical conditions where turning points first appear in the $y_{p}-\epsilon$ curve can be determined from the conditions

$\frac{d \epsilon}{d y_{p}}=\frac{d^{2} \epsilon}{d y_{p}^{2}}=0$

Equations 16 imply that this happens for $\gamma=\gamma^{*}$ with

$\gamma^{*}=4 / \operatorname{erfc}\left(y_{p}^{*}\right)-4 \sqrt{\pi} \exp \left(y_{p}^{* 2}\right)$,

where $y_{p}^{*}$ is the root of

$\left(1+2 y_{p}^{* 2}\right) \operatorname{erfc}\left(y_{p}^{* 2}\right)-\frac{2}{\pi} \exp \left(-2 y_{p}^{* 2}\right)=0$.

The following numerical values are found: $\gamma^{*}=$ $3.6306, \epsilon^{*}=0.3539, y_{p}^{*}=0.3003$, and $y_{m}^{*}=$ -0.005 .

At this point, the one-dimensional asymptotic results may be summarized as follows:

1. For $\gamma<\gamma^{*}$, initially distant flames will approach each other as $\epsilon$ (or the strain rate) is increased, until they merge at the stagnation plane. The condition $y_{p}=y_{m}=0$, when substituted in (16), defines a value of $\epsilon, \epsilon_{\text {ext }}$ say, beyond which no (steady strongly burning) solution exists. This extinction value is given by

$$
\epsilon_{e x t}=\frac{\sqrt{\pi}}{2} \exp \left(\frac{-\gamma}{4}\right) \quad\left(\gamma<\gamma^{*}\right)
$$

Although, strictly speaking, the asymptotic structure of the merged reaction zones need to be reconsidered, it is clear that extinction 


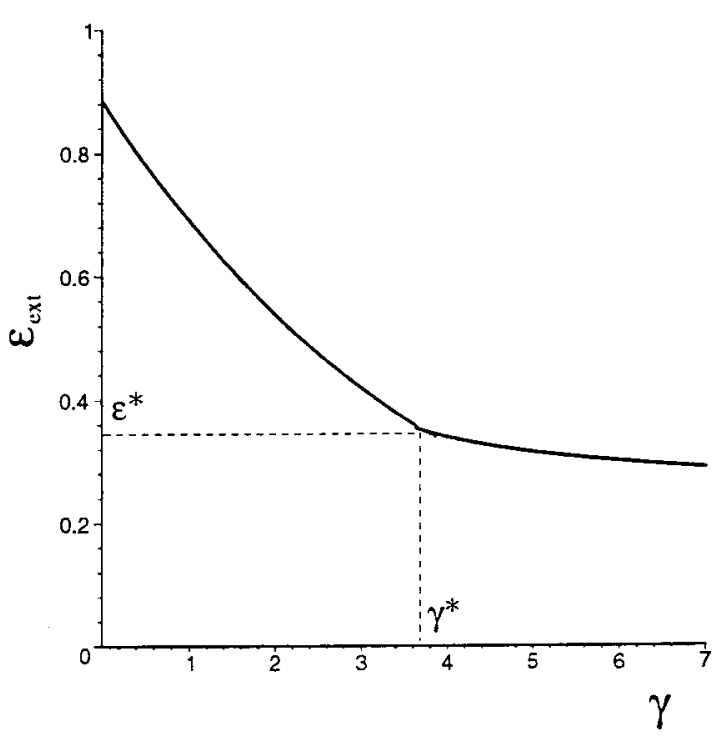

Fig. 4. The value of $\epsilon$ at extinction, $\epsilon_{e x,}$, versus $\gamma$ (solid line).

is associated here with quenching by incomplete combustion.

2. For $\gamma>\gamma^{*}$, similar behavior occurs as $\epsilon$ is increased, but steady strongly burning solutions cease to exist while the flames are at a finite distance from each other, and typically, both in the upper side of the stagnation plane, $y_{p}>y_{m}>0$. The mechanism for extinction here is associated with significant heat being lost from the upper (stronger) flame to sustain the lower flame. Extinction corresponds to $\epsilon=\epsilon_{\text {ext }}$ identified by the turning point in the plot of $y_{p}$ vs. $\epsilon$, where $d \epsilon / d y_{p}=0$. This condition, along with the first two equations in (16) allows the determination of $\epsilon_{e x t}$ and its dependence on $\gamma$. This dependence is obtained in a parametric form $\left\{\epsilon_{e x t}\left(y_{p}\right), \gamma\left(y_{p}\right)\right\}$, where the parameter $y_{p}$ varies in the interval $\left[y_{p}^{*}, \infty\right)$.

Based on the preceding discussion, a plot of $\epsilon_{\text {ext }}$ vs. $\gamma$ is given in Fig. 4. There is a clear distinction at $\gamma=\gamma^{*}$ between the two extinction regimes; the rate of decrease of $\epsilon_{e x t}$ with respect to $\gamma$ is much smaller when $\gamma>\gamma^{*}$. This change appears clearly in Fig. 5, where the location of the upper flame, $y_{p}$, and of the lower, $y_{m}$, are plotted vs. $\gamma$ just prior to extinction. Both locations are equal to zero for $\gamma<\gamma^{*}$, while the distance between them is finite and increases with $\gamma$ for $\gamma>\gamma^{*}$.

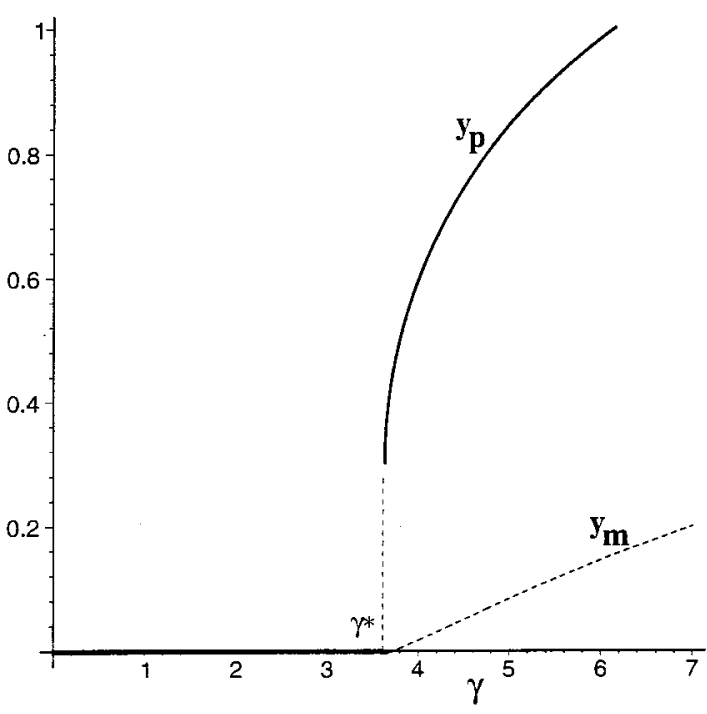

Fig. 5. Locations of the lower and upper flames, $y_{n}$ and $y_{p}$, respectively, versus $\gamma$ just prior to extinction. Both locations are equal to zero for $\gamma<\gamma^{*}$.

The results thus far were obtained in the asymptotic limit of large activation energy. We now present results for finite values of $\beta$. To this end, the one-dimensional equations obtained from (2) by discarding partial derivatives with respect to $x$ are solved numerically with the Arrhenius rate chemistry (4), subject to the boundary conditions (5). In order to relate the numerical results with the asymptotic ones, the parameters $L e_{F}$ and $\Delta_{T}$ are assigned the values one and zero respectively, and results are presented in terms of $\epsilon$ and $\gamma=\gamma_{F}=\beta \Delta_{F}$. Shown in Fig. 6 is a sequence of temperature and reaction rate profiles, corresponding to $\beta=8$, $\gamma=1$, and to three values of $\epsilon$ increasing from top to bottom. It is seen that as the flames approach each other, the stronger flame weakens by supporting the other, initially weaker flame. The two clearly distinguished reaction zones merge near the stagnation plane, as $\epsilon$ approaches the value indicated on the bottom subfigure, and extinguish for a slightly larger value. Figure 7 is similar, except that here $\gamma=5$. Extinction now occurs for a value of $\epsilon$, slightly larger than that of the last subfigure, but without merging of the reaction zones at the stagnation plane. These conclusions are clearly in agreement with the asymptotic results.

Extinction curves similar to the one presented 

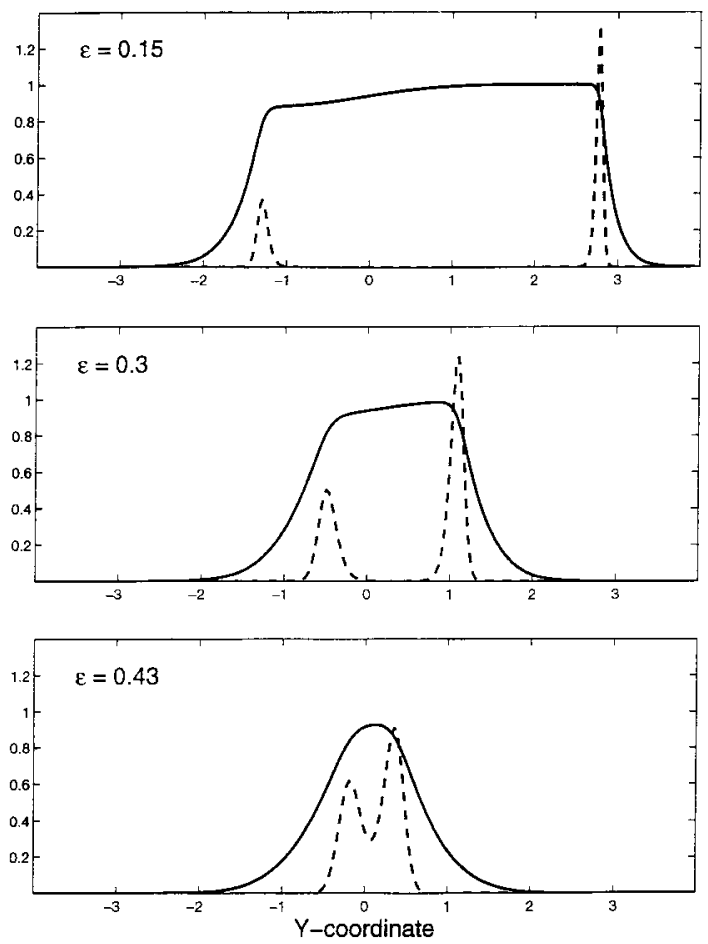

Fig. 6. Temperature (solid-line) and reaction rate profiles (dashed-line), corresponding to $\beta=8, \gamma=1$, and to three values of $\epsilon$. The flames approach each other as $\epsilon$ is increased, until they merge near the stagnation plane. For a value of $\epsilon$ slightly larger than that of the bottom subfigure, the flames extinguish.

in Fig. 4 for $\beta \rightarrow \infty$ are plotted in Fig. 8 for $\beta=8$ and $\beta=20$. The solid line in this figure represents the infinite $\beta$ predictions. It is seen that the trend of the asymptotic results is well reproduced by the numerical calculations, and that the quantitative agreement is improved as $\beta$ is increased. An additional curve is included in this figure, corresponding to the calculations with $\beta=20$ but with $\epsilon_{e x t}$ normalized by its value at $\gamma=0$; the initial decrease clearly reproduces the exponential law given in (18).

\section{THE EDGE-FLAME}

In this section we describe the two-dimensional edge-flame, whose flat trailing wings far downstream have been the object of the last section. We shall begin by the general case where $\epsilon$ is allowed to vary from moderately small up to the extinction values of the planar structure.
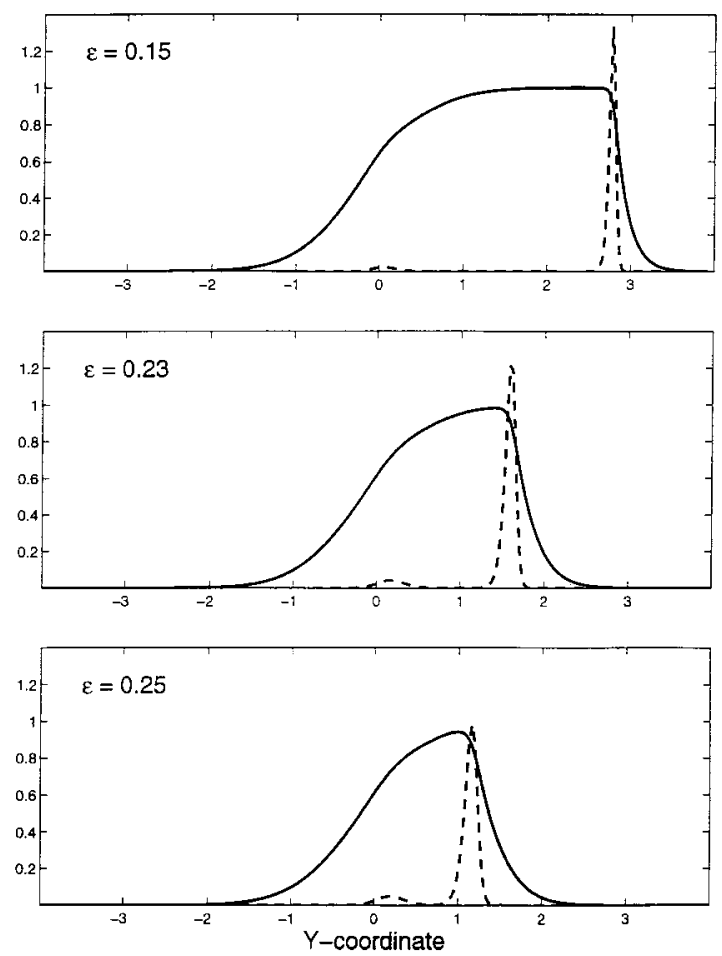

Fig. 7. Similar to Fig. 6, except that $\gamma=5$. Here, extinction occurs, for a value of $\gamma$ slightly larger than that of the bottom subfigure, with the reaction zones being away from the stagnation plane.

The corresponding study is carried out numerically for an Arrhenius chemistry and addresses the influence of $\gamma$ and $\epsilon$ on the propagation speed, $U$, and on the flame shape. This is followed by an analytical description of the numerically difficult small- $\epsilon$ cases where the flame, consisting of the preheat and reaction zones, could be considered thin, say on the scale of its local radius of curvature.

The numerical results presented here are based on Eqs. 2 and boundary conditions (6) and (7). They describe two-dimensional flame fronts which for $U>0$ propagate in the negative $x$-direction, with velocity $U$. The discretized steady equations are obtained by a finite-volume approach and solved using a multigrid method [25]. The eigenvalue $U$ is updated iteratively so that the flame front remains fixed at $x=0$. The numerical grid is rectangular with typically $300 \times 300$ gridpoints distributed nonuniformly over the computational domain. The extent of the latter is $100 \epsilon$ in the $x$-direction and 


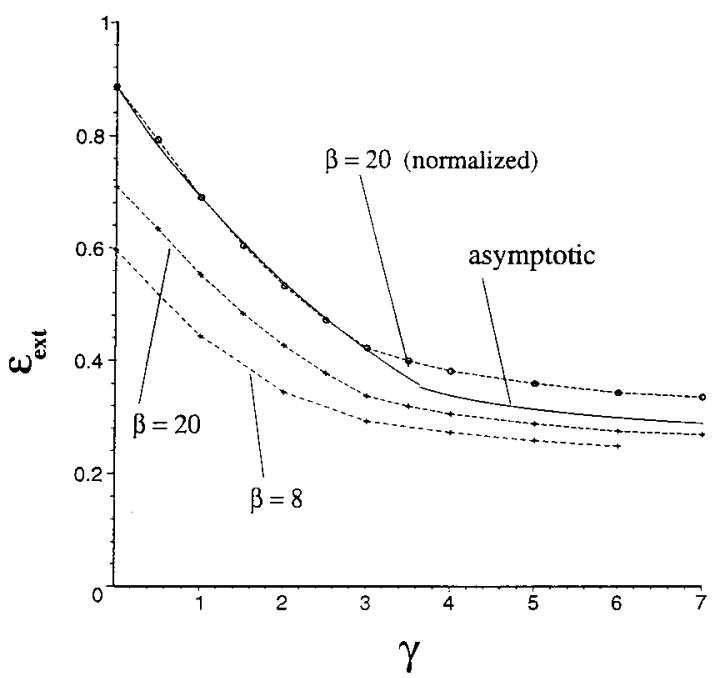

Fig. 8. The value of $\epsilon$ at extinction, $\epsilon_{\text {ext }}$, versus $\gamma$ for the two values $\beta=8$ and $\beta=20$. The solid line is the asymptotic result of Fig. 4. An additional curve corresponding to $\beta=20$ is included but with $\epsilon_{e x t}$ normalized by it value at $\gamma=0$.

10 in the $y$-direction. Note that distances are expressed in terms of the diffusion length $L=$ $\sqrt{2 D_{T} / a}$. The results are presented for $\beta=8$, $L e=1, \alpha_{h}=0.85$, and $\Delta_{T}=0$. The focus is on the influence of the parameters $\gamma=\beta \Delta_{F}$ and $\epsilon$. Nonunity Lewis number effects in the symmetrical case, $\Delta_{F}=\Delta_{T}=0$, can be found in [15].

The dependence of the flame shape on $\epsilon$ and $\gamma$ is summarized in Figs 9, 10, and 11, corresponding respectively to $\gamma=0,1.5$, and 5 . In each figure, temperature contours are plotted on the left corresponding to increasing values of $\epsilon$ from top to bottom; the corresponding reaction rate contours are plotted on the right. We observe that by increasing $\epsilon$ the propagation velocity $U$ varies from a positive value close to unity through zero and then becomes significantly negative. The variation of $U$ from positive to negative, corresponding to flame fronts changing from ignition waves propagating in the negative $x$-direction to extinction waves propagating in the positive $x$-direction, have been found possible for all the values of $\gamma$ considered. Similar conclusions have been drawn previously in studies of edge flames $[11,14,15]$, except when the Lewis numbers were too small. In this latter case, the possibility of having steadily
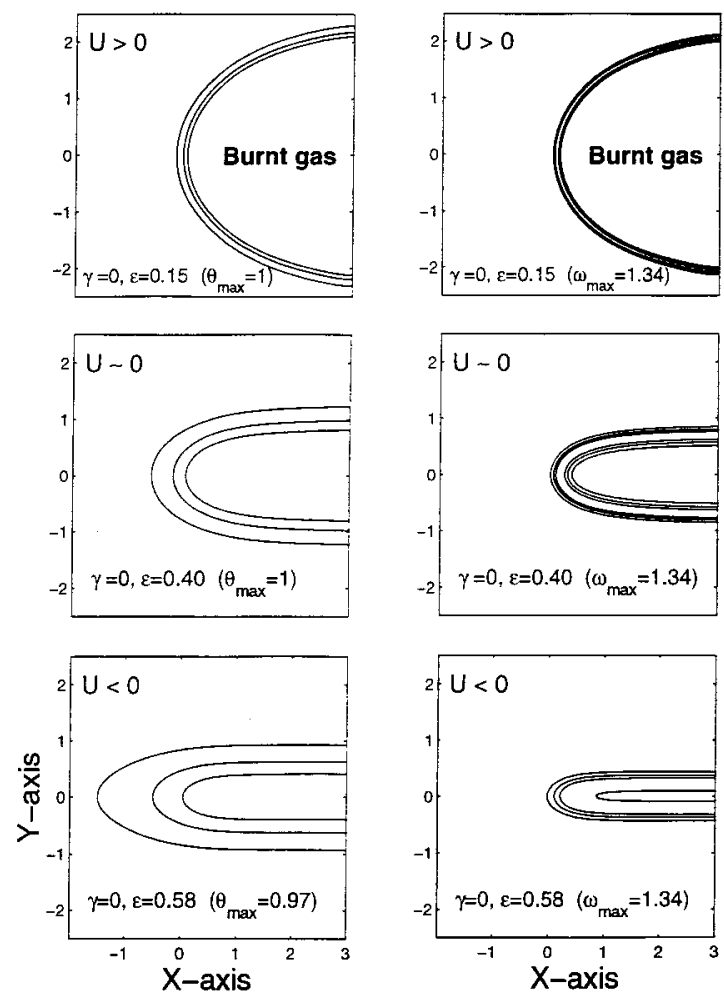

Fig. 9. Temperature and reaction rate contours for the three cases corresponding to $\gamma=0$ and, from top to bottom, to increasing values of $\epsilon$ as indicated. Each of the left subfigures represents three temperature isocontours distributed between zero and the maximum temperature, $\theta_{\text {max }}$. Each of the right subfigures represents four reaction-rate isocontours distributed between zero and the maximum value, $\omega_{\max }$. Depending on $\epsilon$ the front evolves from an ignition front, $U>0$, to an extinction front, $U<0$ (from top to bottom). The values of other parameters are $l_{F}=0$, $\beta=8$, and $\alpha_{h}=0.85$

retreating extinction fronts is typically suppressed. The figures also show that an increase in the value of $\gamma$ changes the flame from a symmetrical tongue-shaped to a hook-shaped form. This is accompanied by (i) an increase of the front curvature and (ii) a shift of the leading edge away from the stagnation plane. These two effects, leading to a decrease in propagation speed will be also revealed in the asymptotic small- $\epsilon$ study given below. In Fig. 12 the propagation speed $U$ is plotted versus (the square root of the strain rate) $\epsilon$ for selected values of $\gamma$. The graph clearly identifies regions where the edge flame advances $(U>0)$ from regions where it retracts $(U<0)$. For small $\epsilon$, the flame 

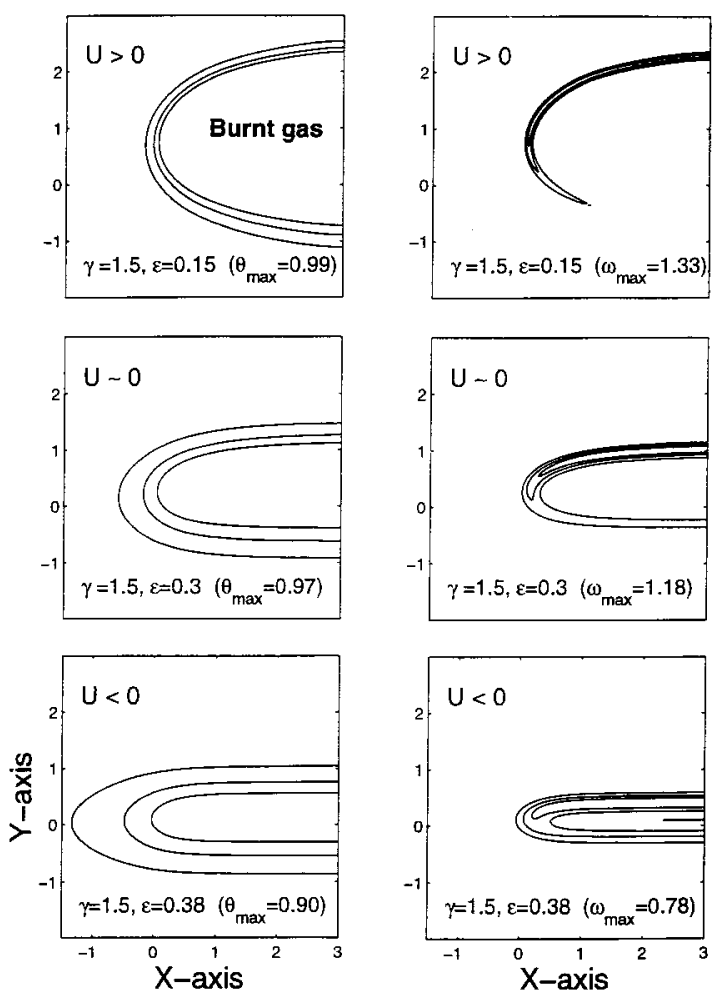

Fig. 10. Same as Fig. 9, but with $\gamma=1.5$.

always propagates forwards at a speed near the laminar flame speed. As $\epsilon$ increases the flame slows down and eventually moves backwards until it extinguishes at $\epsilon=\epsilon_{\text {ext }}$ - the value of $\epsilon$ furthest to the right for each curve. The range of $\epsilon$ for which burning is possible reduces with increasing $\gamma$. For small $\gamma$ the extinction fronts $(U<0)$ travel at significant speeds, of the magnitude of the laminar flame speed, or higher. For larger values of $\gamma$ extinction fronts are also possible but their speed is relatively low. ${ }^{1}$

For small $\epsilon$, it is possible to derive an analytical approximate solution for the shape and speed of the edge flame. The methodology follows that detailed in Matalon and Matkowsky [18]. We start with the large activation energy formulation in the limit expressed in (9), resulting in the equations

\footnotetext{
${ }^{1}$ Note that we have also included in the left of Fig. 12 three plots of $U$ vs. $\epsilon$ according to the asymptotic expressions to be derived below.
}
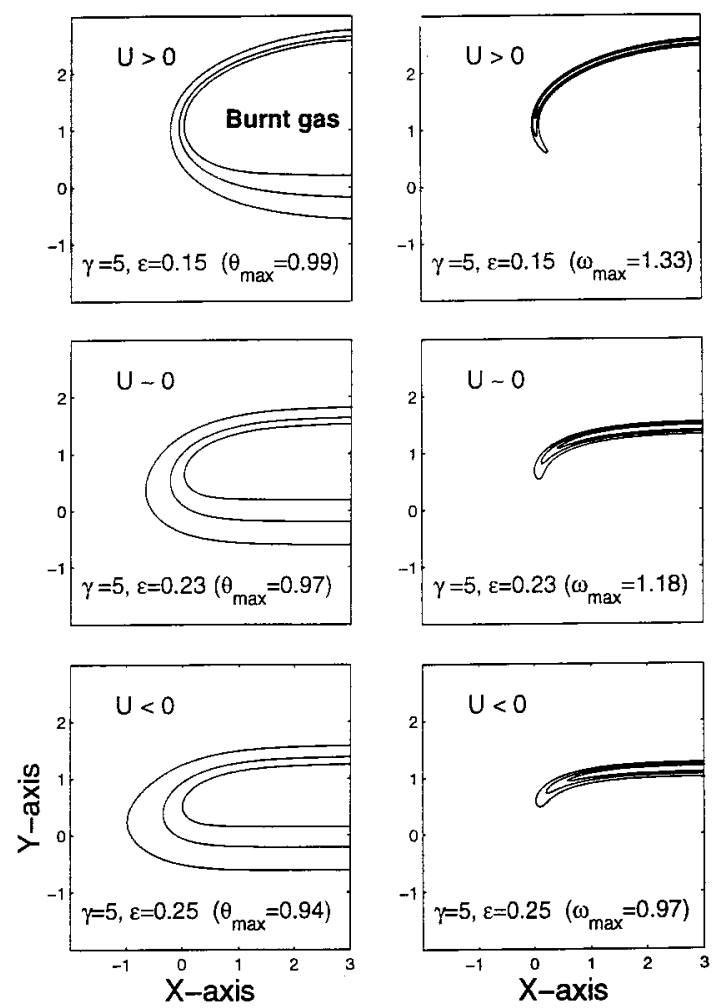

Fig. 11. Same as Fig. 9, but with $\gamma=5$.

$U \frac{\partial \theta^{0}}{\partial x}-2 \epsilon y \frac{\partial \theta^{0}}{\partial y}=\epsilon \Delta \theta^{0}$

$U \frac{\partial h}{\partial x}-2 \epsilon y \frac{\partial h}{\partial y}=\epsilon \Delta h+\epsilon l_{F} \Delta \theta^{\circ}$

that must be satisfied on either side of the reaction sheet. Here $\theta^{0}$ is the leading order temperature (in an expansion in terms of $\beta^{-1}$ ) and $h$ is the enthalpy perturbation defined in this section as $h=\theta^{1}+y_{F}^{1}-\frac{\gamma}{2} \operatorname{erfc}(y)$. Note that $h$ thus defined appears as the excess enthalpy $\theta^{1}+y_{F}^{1}$ from which we have subtracted its (frozen) value at $x=-\infty$. The boundary conditions are

$\theta^{0}=h=0$ as $x \rightarrow-\infty$ or $|y| \rightarrow \infty$

$\frac{\partial \theta^{0}}{\partial x}=\frac{\partial h}{\partial x}=0$ as $x \rightarrow \infty$

Finally, the jump conditions that must be satisfied across the reaction sheet are 


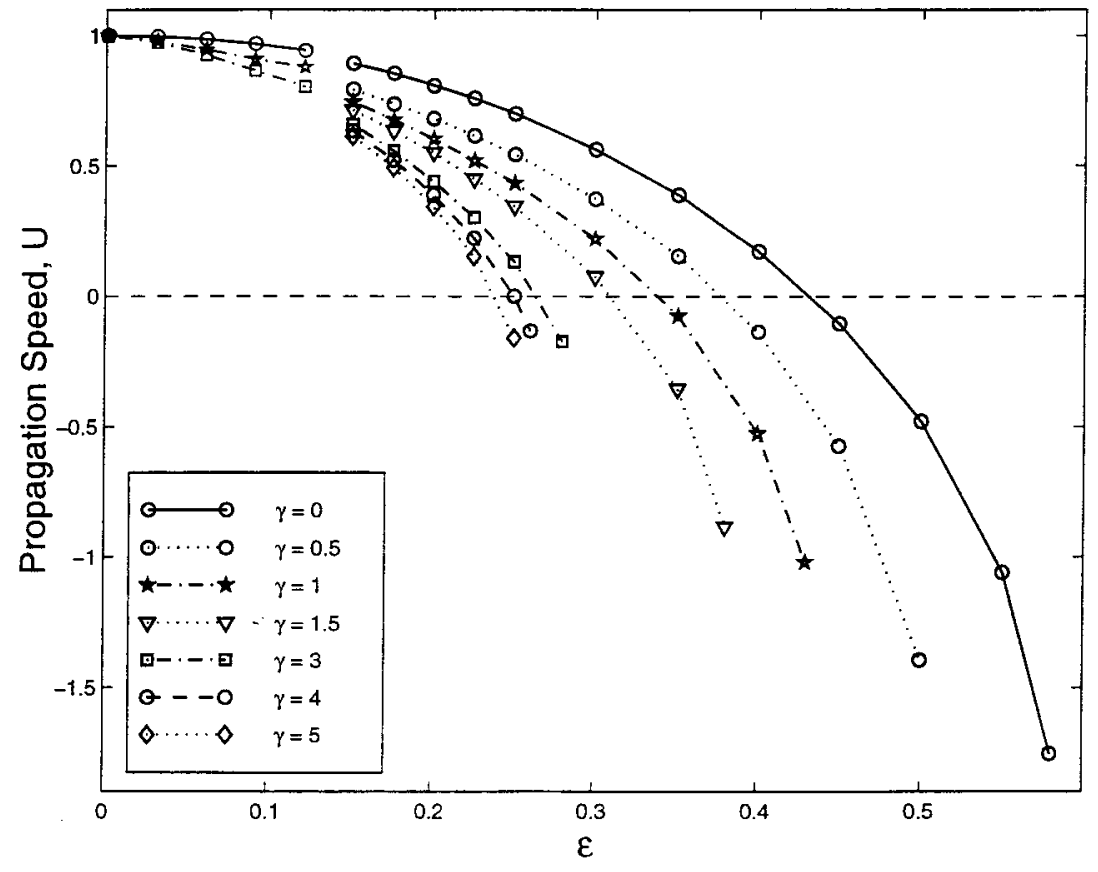

Fig. 12. Propagation speed $U$ versus $\boldsymbol{\epsilon}$ for several values of $\gamma . U$ has been normalized by the planar (unstretched) flame speed corresponding to a uniform mixture, $\gamma=0$. For each $\gamma$, the upper bound for the existence of the $2 D$ fronts is $\epsilon_{\text {exi }}$, the extinction value of the pair of flat flames given in Fig. 8 (and corresponding to $\beta=$ 8). The curves for small $\epsilon$ (originating from the point $[\epsilon=0, U=1]$ ) are based on the asymptotic results.

$$
\begin{aligned}
& {\left[\theta^{(0}\right]=[h]=0} \\
& -\frac{1}{l_{F}}\left[\frac{\partial h}{\partial n}\right]=\left[\frac{\partial \theta^{0}}{\partial n}\right]=-\epsilon^{-1} S_{0}^{2} e^{\sigma / 2}
\end{aligned}
$$

where $S_{0}=\exp \{-\gamma / 4 \operatorname{erfc}(y)\}, n$ a coordinate along the normal to the reaction sheet increasing from the unburnt side to the burnt side, and $\sigma$ the value of $h$ at the reaction sheet.

The asymptotic treatment for small $\epsilon$ necessitates analysis of the preceding problem both on the scale $L=\sqrt{2 D_{T} / a}$, used as unit length up to now, and on the scale $L / \epsilon \sim S_{L}^{0} / a$ which represents the standoff distance between the planar wings. However, as we shall see, the results based on the latter scale, i.e., for order unity values of the scaled coordinates $\tilde{x}=\epsilon x$ and $\tilde{y}=\epsilon y$, allow one to obtain a uniformly valid description of the flame front. Hence, for simplicity, the derivation below will be presented in terms of the coordinates $(\tilde{x}, \tilde{y})$. On either scale, diffusion effects are small except in a thin flame zone which coincides with the reaction sheet $\tilde{x}=f(\tilde{y})$ as $\epsilon \rightarrow 0$. In the outer regions, on both sides of the flame, we have

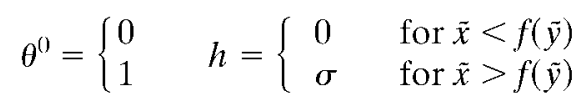

which are also the matching conditions for the inner solutions that we shall construct next. We note that a unit normal vector to the flame (pointing to the burnt gas) is given by

$\mathbf{n}=\frac{\mathbf{i}-f^{\prime}(\tilde{y}) \mathbf{j}}{\sqrt{1+f^{\prime}(\tilde{y})^{2}}}$,

so that the flame speed, defined as usual relative to the fresh mixture, is given by

$S_{f}=(U \mathbf{i}-2 \bar{y} \mathbf{j}) \cdot \mathbf{n}=\frac{U+2 \tilde{y} f^{\prime}(\tilde{y})}{\sqrt{1+f^{\prime}(\tilde{y})^{2}}}$

We introduce a coordinate system attached to the flame, stretched appropriately, by writing

$\zeta=\{\bar{x}-f(\tilde{y})\} \epsilon^{-2}, \quad \tilde{y}=\tilde{y}$,

so that $\zeta=0$ represents the reaction sheet. We then introduce expansions in the form

$$
\begin{aligned}
& f=f_{0}+\epsilon^{2} f_{1}+\ldots, \\
& U=U_{0}+\epsilon^{2} U_{1}+\ldots, \\
& \sigma=\sigma_{0}+\epsilon^{2} \sigma_{1}+\ldots
\end{aligned}
$$

and similar expressions for $\theta^{()}$and $h$. Note that $S_{0}$ is regarded as an arbitrary $\mathrm{O}(1)$ function; its 
actual form will be reinstated after the final expansions have been obtained. We proceed now in solving for $\theta_{0}, h_{0}, \theta_{1}$, and $h_{1}$. To leading order we have

$$
\begin{aligned}
& \left(U_{0}+2 \tilde{y} f_{0}^{\prime}\right) \frac{\partial \theta_{0}}{\partial \zeta}-\left(1+f_{0}^{\prime 2}\right) \frac{\partial^{2} \theta_{0}}{\partial \zeta^{2}}=0 \\
& \left(U_{0}+2 \tilde{y} f_{0}^{\prime}\right) \frac{\partial h_{0}}{\partial \zeta}-\left(1+f_{0}^{\prime 2}\right) \frac{\partial^{2} h_{0}}{\partial \zeta^{2}} \\
& =l_{F}\left(1+f_{0}^{\prime 2}\right) \frac{\partial^{2} \theta_{0}}{\partial \zeta^{2}}
\end{aligned}
$$

Consequently, $\theta_{0}$ and $h_{0}$ are given by

$\theta_{0}= \begin{cases}\exp (\alpha \zeta) & \text { for } \zeta<0 \\ 1 & \text { for } \zeta>0\end{cases}$

$h_{0}= \begin{cases}-\alpha l_{F} \zeta \exp (\alpha \zeta) & \text { for } \zeta<0 \\ \sigma_{0}=0 & \text { for } \zeta>0\end{cases}$

with $\alpha=\left(U_{0}+2 \tilde{y} f_{0}^{\prime}\right) /\left(1+f_{0}^{\prime 2}\right)$, so as to be continuous at $\zeta=0$ and satisfy the matching requirement $(23)$ as $|\zeta| \rightarrow \infty$. The remaining jump relations provide the flame speed as $S_{f 0}=$ $S_{0}$. Thus, to leading order, the flame speed is locally the speed of a planar flame at the conditions prevailing at $\tilde{y}$.

In the next approximation we have to solve

$$
\begin{gathered}
\left(U_{0}+2 \tilde{y} f_{0}^{\prime}\right) \frac{\partial \theta_{1}}{\partial \zeta}-\left(1+f_{0}^{\prime 2}\right) \frac{\partial^{2} \theta_{1}}{\partial \zeta^{2}} \\
=\mathscr{L}\left(\theta_{0}\right)-\left(U_{1}+2 \tilde{y} f_{1}^{\prime}\right) \frac{\partial \theta_{0}}{\partial \zeta}+2 \tilde{y} \frac{\partial \theta_{0}}{\partial \tilde{y}} \\
\left(U_{0}+2 \tilde{y} f_{0}^{\prime}\right) \frac{\partial h_{1}}{\partial \zeta}-\left(1+f_{0}^{\prime 2}\right) \frac{\partial^{2} h_{1}}{\partial \zeta^{2}} \\
=\mathscr{L}\left(h_{0}+l_{F} \theta_{0}\right)-\left(U_{1}+2 \tilde{y} f_{1}^{\prime}\right) \frac{\partial h_{0}}{\partial \zeta} \\
+l_{F}\left(1+f_{0}^{\prime 2}\right) \frac{\partial^{2} \theta_{1}}{\partial \zeta^{2}}+2 \tilde{y} \frac{\partial h_{0}}{\partial \tilde{y}}
\end{gathered}
$$

where the operator $\mathscr{L}$ is defined by

$\mathscr{L} \equiv 2 f_{0}^{\prime} f_{1}^{\prime} \frac{\partial^{2}}{\partial \zeta^{2}}-f_{0}^{\prime \prime} \frac{\partial}{\partial \zeta}-2 f_{0}^{\prime} \frac{\partial^{2}}{\partial \tilde{y} \partial \zeta}$

For $\theta_{1}$ and $h_{1}$ to be bounded and match the outer solution (23), we must have

$\theta_{1}=0$ and $h_{1}=\sigma_{1}$ for $\zeta \geq 0$
Integration of (27) from $\zeta=-\infty$ to $\zeta=0^{-}$yields

$$
\begin{aligned}
\left(1+f_{0}^{\prime 2}\right)\left[\frac{\partial \theta_{1}}{\partial \zeta}\right]= & I_{\theta}-\left(U_{1}+2 \tilde{y} f_{1}^{\prime}\right)-2 \tilde{y} \frac{\alpha^{\prime}}{\alpha^{2}} \\
\left(U_{0}+2 \tilde{y} f_{0}^{\prime}\right) \sigma_{1}+ & \left(1+f_{0}^{\prime 2}\right)\left[\frac{\partial h_{1}}{\partial \zeta}\right] \\
= & -l_{F}\left(1+f_{0}^{\prime 2}\right)\left[\frac{\partial \theta_{1}}{\partial \zeta}\right] \\
& +I_{h}+l_{F} I_{\theta}-2 \tilde{y} \frac{\alpha^{\prime}}{\alpha^{2}} l_{F}
\end{aligned}
$$

Here $\alpha^{\prime}$ is the derivative of $\alpha$ with respect to $\tilde{y}$ and the quantities $I_{\theta}$ and $I_{h}$ are given by

$$
\begin{aligned}
& I_{\theta}=\int_{-\infty}^{0} \mathscr{L}\left(\theta_{0}\right) d \zeta=2\left(U_{0}+2 \tilde{y} f_{0}^{\prime}\right) \frac{f_{0}^{\prime} f_{1}^{\prime}}{1+f_{0}^{\prime 2}}-f_{0}^{\prime \prime} \\
& I_{h}=\int_{-\infty}^{0} \mathscr{L}\left(h_{0}\right) d \zeta=-2 l_{F}\left(U_{0}+2 \tilde{y} f_{0}^{\prime}\right) \frac{f_{0}^{\prime} f_{1}^{\prime}}{1+f_{0}^{\prime 2}}
\end{aligned}
$$

When these expressions are substituted in Eqs. 29 , and use is made of the jump conditions, we obtain

$$
\begin{gathered}
\left(U_{0}+2 \tilde{y} f_{0}^{\prime}\right) \sigma_{1}=-l_{F}\left(f_{0}^{\prime \prime}+2 \tilde{y} \frac{\alpha^{\prime}}{\alpha^{2}}\right) \\
\left(U_{0}+2 \tilde{y} f_{0}^{\prime}\right) \frac{f_{0}^{\prime} f_{1}^{\prime}}{1+f_{0}^{\prime 2}}-\left(U_{1}+2 \tilde{y} f_{1}^{\prime}\right)= \\
\left(1+\frac{l_{F}}{2}\right)\left(f_{0}^{\prime \prime}+2 \tilde{y} \frac{\alpha^{\prime}}{\alpha^{2}}\right),
\end{gathered}
$$

which determine $\sigma_{1}$ and $S_{f 1}$. Combining these with the leading order results we obtain after some manipulations

$$
S_{f} \sim S_{0}\left[1-\left(1+\frac{l_{F}}{2}\right)\left(\frac{U_{0} f_{0}^{\prime \prime}}{1+f_{0}^{\prime 2}}+2 \tilde{y} \frac{S_{0}^{\prime}}{S_{0}}\right) \frac{\epsilon^{2}}{S_{0}^{2}}\right]
$$

for the flame speed and

$$
\theta_{F} \sim 1+\frac{\ln S_{0}^{2}}{\beta}-\frac{l_{F}}{\beta}\left(\frac{U_{0} f_{0}^{\prime \prime}}{1+f_{0}^{\prime 2}}+2 \tilde{y} \frac{S_{0}^{\prime}}{S_{0}}\right) \epsilon^{2}
$$

for the flame temperature.

In the homogeneous case corresponding to $\gamma=0$ we have $S_{0}=1$, so that (31) leads after using (24) to 


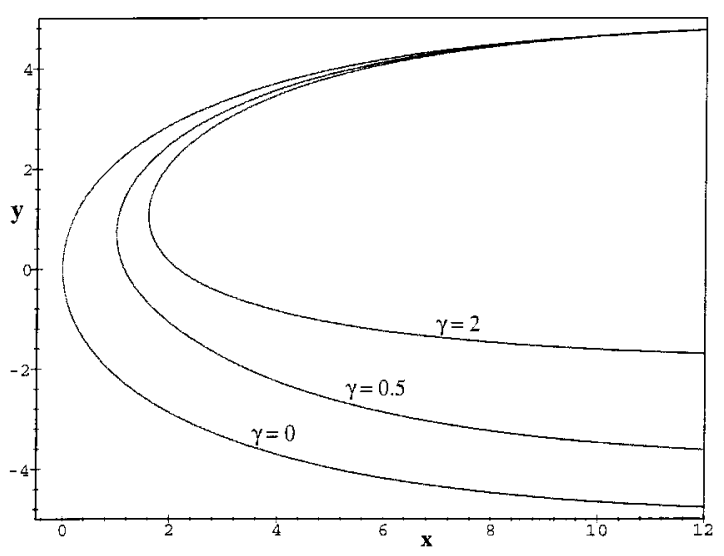

Fig. 13. Flame shape for $\epsilon=0.1$ and for three values of $\gamma$, based on the asymptotic expression (35).

$\frac{U_{0}+2 \tilde{y} f_{0}^{\prime}}{\sqrt{1+f_{0}^{\prime 2}}}=1$

By applying this relation at the leading edge, located at $\tilde{y}=0$ by symmetry, we obtain $U_{0}=$ 1 ; hence

$f_{0}^{\prime}=\frac{4 \tilde{y}}{1-4 \tilde{y}^{2}}$ and $f_{0}=-\frac{1}{2} \ln \left(1-4 \tilde{y}^{2}\right)$

(see Fig. 13). Then by reusing (31), the correction due to stretch can be computed yielding

$S_{f}=1-4 \epsilon^{2}\left(1+\frac{l_{F}}{2}\right) /\left(1+4 \tilde{y}^{2}\right)$ and

$U=1-4 \epsilon^{2}\left(1+\frac{l_{F}}{2}\right)$,

as found in [20].

To determine the propagation speed $U$ in the nonhomogeneous case we analyze the relation (31). We note that viewed on the $\tilde{y}$-scale, $S_{0}$ is a piecewise constant function with a sharp transition near $\tilde{y} \approx 0$, more properly described on the $y$-scale. It could be verified however that, when expressed in terms of $y$, Eq. 33 provides a uniformly valid description of the flame front. To leading order we thus have

$\frac{U_{0}+2 \epsilon y f_{0}^{\prime}}{\sqrt{1+f_{0}^{\prime 2}}}=S_{0}, \quad S_{0}=\exp \left\{-\frac{\gamma}{4} \operatorname{erfc}(y)\right\}$

which is an algebraic equation for the flame slope $f_{0}^{\prime}$,
$\left(S_{0}^{2}-4 \epsilon^{2} y^{2}\right) f_{0}^{\prime 2}-4 \epsilon y U_{0} f_{0}^{\prime}+\left(S_{0}^{2}-U_{0}^{2}\right)=0$,

the roots of which are

$f_{0}^{\prime}=\frac{2 \epsilon y U_{0} \pm \sqrt{\Delta}}{S_{0}^{2}-4 \epsilon^{2} y^{2}}$,

$\Delta=\left(U_{0}^{2}+4 \epsilon^{2} y^{2}-S_{0}^{2}\right) S_{0}^{2}$

There are two locations where the slope becomes infinite and the flame front horizontal:

(1) as $y \rightarrow y_{p} \equiv \frac{1}{2 \epsilon}$

$$
f_{0}^{\prime}=\frac{2 \epsilon y U_{0}+\sqrt{\Delta}}{S_{0}^{2}-4 \epsilon^{2} y^{2}} \rightarrow+\infty
$$

(2) as $y \rightarrow y_{m} \equiv-\frac{e^{-\gamma / 2}}{2 \epsilon}$

$$
f_{0}^{\prime}=\frac{2 \epsilon y U_{0}-\sqrt{\Delta}}{S_{0}^{2}-4 \epsilon^{2} y^{2}} \rightarrow-\infty
$$

The edge-flame asymptotes far downstream to the positions $y_{p}$ and $y_{m}$ identified previously as the locations of the planar fronts in the limit $\epsilon \rightarrow 0$; see Eqs. 17. The flame front is therefore described by one or the other root of (34) connected smoothly at some location, say $y_{S}$. The requirement that $f_{0}^{\prime}$ and $f_{0}^{\prime \prime}$ are continuous at $y_{S}$ implies that

$U_{0}^{2}+4 \epsilon^{2} y_{S}^{2}-S_{0}^{2}\left(y_{S}\right)=0$ and $\left.\frac{d S_{0}^{2}}{d y}\right|_{y=y_{S}}=8 \epsilon^{2} y_{S}$

hence

$$
\begin{aligned}
& \epsilon^{2}=\frac{\gamma}{8 \sqrt{\pi}} \frac{\exp \left(-y_{S}^{2}\right)}{y_{S}} \exp \left\{-\frac{\gamma}{2} \operatorname{erfc}\left(y_{S}\right)\right\} \\
& U_{0}^{2}=\exp \left\{-\frac{\gamma}{2} \operatorname{erfc}\left(y_{S}\right)\right\}-4 \epsilon^{2} y_{S}^{2}
\end{aligned}
$$

These last two equations could be viewed, for fixed $\gamma$, as a parametric representation of the form $\left\{\epsilon\left(y_{S}\right), U_{0}\left(y_{S}\right)\right\}$. Thus, for any given $\gamma$ and $\epsilon$, a unique solution $\left(U_{0}, y_{S}\right)$ is found, provided that $\epsilon$ is sufficiently small. For illustration, Fig. 13 depicts the flame shape for $\epsilon=0.1$ and for three values of $\gamma$. This is done by integration of (35), after using (36) to deter- 
mine $U_{0}$ and $y_{S}$. From (37) one obtains for $\epsilon \rightarrow$ 0

$y_{S}^{2} \sim \frac{1}{2} L\left(\frac{\gamma^{2}}{32 \pi \epsilon^{4}}\right)$,

$U_{0}^{2} \sim 1-2 \epsilon^{2} L\left(\frac{\gamma^{2}}{32 \pi \epsilon^{4}}\right)$ for $\epsilon \rightarrow 0$,

where $\mathrm{L}$ is a slowly increasing function defined by $\mathrm{L}(u) \exp (\mathrm{L}(u))=u$. $\mathrm{L}(u)$ is often known as the Lambert W function; it satisfies $\mathrm{L}(0)=0$ and $\mathrm{L}(u) \sim \ln (u)$ as $u \rightarrow \infty$. An important quantity to be used below is the location of the leading edge of the flame, say $y_{N}$. In first approximation, it is defined as the location where $f_{0}^{\prime}$ is zero, namely by $S_{0}\left(y_{N}\right)=U_{0}$, so that

$y_{N}=\operatorname{erfc}^{-1}\left\{\frac{-4 \ln \left(U_{0}\right)}{\gamma}\right\}$

Note that the preceding equation implies that for finite $\gamma$ the leading edge is located far from the stagnation plane (more precisely, we have $y_{N} \rightarrow \infty$ but $\tilde{y}_{N} \rightarrow 0$ for $\epsilon \rightarrow 0$ ). Also, the flame curvature at the leading edge is found to be

$f_{0}^{\prime \prime}\left(\tilde{y}_{N}\right)=\frac{\gamma}{4 \sqrt{\pi}} \frac{\exp \left(-y_{N}^{2}\right)}{y_{N}} U_{0} \sim L\left(\frac{\gamma^{2}}{32 \pi \epsilon^{4}}\right)$

The propagation speed $U$ could now be calculated using (31) leading to the approximation:

$$
\begin{aligned}
U \sim & \left\{1-2 \epsilon^{2} L\left(\frac{\gamma^{2}}{32 \pi \epsilon^{4}}\right)\right\} \\
& +\left(1+\frac{l_{F}}{2}\right) \epsilon^{2} L\left(\frac{\gamma^{2}}{32 \pi \epsilon^{4}}\right)
\end{aligned}
$$

The deviation of $U$ from one included in the curly bracket translates the fact that the edge is located in a region where the mixture enthalpy is slightly less than its value at infinity. The following term accounts for flame curvature and differential diffusion. Thus, an increase in $\gamma$ affects $U$ by shifting the location of the flame leading edge away from the stagnation plane and by increasing flame curvature. For small $\epsilon$, these two effects are of the same order of magnitude.

\section{CONCLUSION}

We have studied in the counterflow configuration the effect of unequal feed conditions on flame propagation. The opposed fresh streams consisted of an off-stoichiometric combustible mixture with unequal enthalpies. In the resulting nonuniform mixture, we have addressed two particular types of burning solutions. The first type corresponds to a pair of flat flames of unequal strength squeezed against each other by the flow. Their coupled behavior has been described analytically and numerically under intense burning conditions up to near-extinction conditions. Special attention has been devoted to the dependence of the extinction regimes on the difference in the feed conditions. The second type corresponds to a (two-dimensional) edge-flame propagating transversely to the plane of the flow. These fronts have been described numerically for different degrees of nonuniformity in the enthalpy and under a wide range of flow conditions. In particular, the variation of their propagation speeds from positive to negative in terms of the strain rate has been determined. For small values of the strain rate an analytical description has been provided which complements the numerical findings.

This work has been partially supported by the National Science Foundation under Grants CTS9521022 and DMS9703716.

\section{REFERENCES}

1. Phillips, H., Tenth Symposium (Intemational) on Combustion, The Combustion Institute, 1965, p. 1277-1283.

2. Ohki, Y., and Tsuge, S., in Dynamics of Reactive Systems, Part I (J. R. Bowen, J. C. Leyer, and R. I. Soloukhin, Eds.), Progress in Astronautics and Aeronautics Vol. 105, 1986, p. 233-245.

3. Dold, J. W., Combust. Flame 76:71 (1989).

4. Hartley, L. J., and Dold, J. W., Combust. Sci. Technol. $80: 23$ (1991).

5. Liñán, A., in Combust. in High Speed Flows (J. Buckmaster, T. L. Jackson, and A. Kumar, Eds.), Kluwer Academic, Boston, 1994, p. 461.

6. Kioni, P. N., Rogg, B., Bray, C., and Liñán, A., Combust. Flame 95:276-290 (1993).

7. Buckmaster, J., and Matalon, M., Twenty-Second Symposium (Intemational) on Combustion, The Combustion Institute, Pittsburgh, 1988, pp. 1527-1535.

8. Ruetsch, G. R., Vervisch, L., and Liñán, A., Phys. Fluids 7(6):1447-1454 (1995). 
9. Daou, J., and Liñán, A., Combust. Theory Modelling 2:449-477 (1998).

10. Shay, M. L., and Ronney, P. D., Combust. Flame 112:171 (1998).

11. Buckmaster, J., Combust. Sci. Technol. 115:41 (1996).

12. Vedarajan, T. G., and Buckmaster, J. D., Combust. Flame 114:267 (1998).

13. Vedarajan, T. G., Buckmaster, J. D., and Ronney, P., Twenty-Seventh Symposium (Intemational) on Combustion, The Combustion Institute, Pittsburgh, 1999.

14. Buckmaster, J., and Short, M., Combust. Theory Modelling 3:199-214 (1999).

15. Daou, J., and Liñán, A., Combust. Flame 118:479-488 (1999).

16. Liu, J. B., and Ronney, P. D., Premixed edge-flames in spatially-varying straining flows, Combust. Sci \& Technol 144:21-46 (1999).

17. Clavin, P., and Williams, F. A., JFM 116:251-282 (1982).

18. Matalon, M., and Matkowsky, B. J., JFM 124:239-259 (1982).
19. Westbrook, C. K., Acta Astronautica 5:1185-1198 (1978).

20. Mikolaitis, D. W., Combust. Flame 57:87 (1984).

21. Bush, W. B., Fendell, E. F., and Stanton, F. F., Combust. Sci. Technol. 24:53 (1980).

22. Buckmaster, J., and Ludford, G. S., Lectures on Mathematical Combustion, SIAM Press, Philadelphia, 1983.

23. Sato, J., and Tsuji, H., Combust. Sci. Technol. 33:193 (1983).

24. Zeldovich, Y. B., Barenblatt, G. I., Librovich, V. B., and Makhviladze, G. M., The Mathematical Theory of Combustion and Explosions, Consultants Bureau, New York, 1985, p. 332.

25. Ruge, J., and Stüben, K. (1983). Proceedings of Multigrid Conference, Bristol. 